\title{
Load Balancing in Multi-Cloud with Performance Analysis
}

\author{
Akankhya Gogoi, Mr. A. M. J. Muthukumaran
}

\begin{abstract}
Cloud computing enables distributed file system mainly for large scale data storage and data access. The main character is that the contents of the file system can be stored in multiple machines which transfer data through the internet with robustness and scalability. In this paper, we explore the scenario to deploy a computing cluster on the top of a multi cloud infrastructure, for solving Many Task Computing (MTC) applications. For efficient handling of multiple tasks, need to have the performance analysis and optimization of all tasks in the multi-cloud environment. Performance analyses consist of CPU scheduling, Memory utilization, I/O tasks, and cost benefits. In the method, Job is allocated by front end server and service LAN is used. This research will achieve the process of multitasking environment thus balancing the load and ensures the reliability of the system.

Index Terms: Cloud, Load balance, Multi cloud infrastructure, Multi task computing.
\end{abstract}

\section{INTRODUCTION}

Cloud computing is an on demand service in which shared resources, information, software and other devices are provided according to the clients requirement at specific time. It's a term which is generally used in case of Internet. The whole Internet can be viewed as a cloud. Capital and operational costs can be cut using cloud computing. In spite of its enormous advantages, single cloud environment is not efficient for Many-task computing in order to handle the parallel processing of MTC Applications. Single-Cloud architecture does not minimize the sharing of hardware, memory, resources and I/O Response and Cost optimizations. The main aim of the MTC Application is to implement the High Performance Computing characters in the parallel processing [1].

A common way to mitigate risk is to employ redundant independent systems. Public cloud users can achieve this by using two or more different cloud providers. Public cloud has both technical and business risk. Technical risks such as outages and other service failures are often at the forefront of IT concerns. Working with multiple cloud provider help mitigate the risk of service disruption in local data center.

Many Task Computing (MTC) defines different types of high-performance applications involving many different tasks, and requires large number of computational resources over short periods of time. These tasks can differ in size and nature, loosely coupled or tightly coupled, or compute-intensive or data intensive. Cloud computing technologies can offer important benefits for IT organizations and data centers. They help in running MTC applications with elasticity and rapid provisioning and enable the organization to increase or decrease its infrastructure capacity within minutes and it can reduce or even eliminate their in-house infrastructures. Thus it results on a reduction in capital investment and personnel costs with different hardware configurations, operating systems, and software packages [3].

This paper is organized as follows. In section II, problem definition is discussed. In section III, frame work of the proposed system is discussed. Techniques used for the proposed system is discussed in section IV followed by results in section $\mathrm{V}$.

\section{Problem Definition}

The popular file system for networked computers is the Network File System (NFS). It is a way to share files between machines on a network as if the files were located on the client's local hard drive. The machines are required to be under a common administrator and be able to communicate securely. The first one is that it depends on a single central node to manage almost all operations of every data block in the file system. As a result it can be a bottleneck resource and a single point of failure [4].

Akankhya Gogoi is with Department of Computer Science and Engineering, SRM University, Kattankulathur - 603 203, Tamil Nadu, India (e-mail: akankhyag@ gmail.com, Phone number- 7299809380).

Mr. A. M. J. Muthukumaran is an assistant professor in Department of Computer Science and Engineering, SRM University, Kattankulathur - 603203, Tamil Nadu, India (e-mail:muthuamj@yahoo.com, Phone number-9840788878). 


\section{Framework Of Proposed System}

Multi-cloud strategy is the concomitant use of two or more cloud services. It minimizes the risk of data loss or downtime due to a localized component failure in a cloud computing environment. Such a failure can occur in hardware, software, or infrastructure. A multi cloud strategy meet the need of the diverse needs of different customers by avoiding "vendor lock in" that is a service that ensures customer dependence on the vendor service and provides different infrastructure to the customers. A multi cloud environment can attract traffic from different customers and partners through the fastest possible network. It offers hardware, software and infrastructure redundancy necessary to optimize fault tolerance. Some clouds can perform a task better than others. For example, a certain cloud might handle large numbers of requests per unit time requiring small data transfers on the average, but a different cloud might perform better for smaller numbers of requests per time involving large data transfers on the average [5].

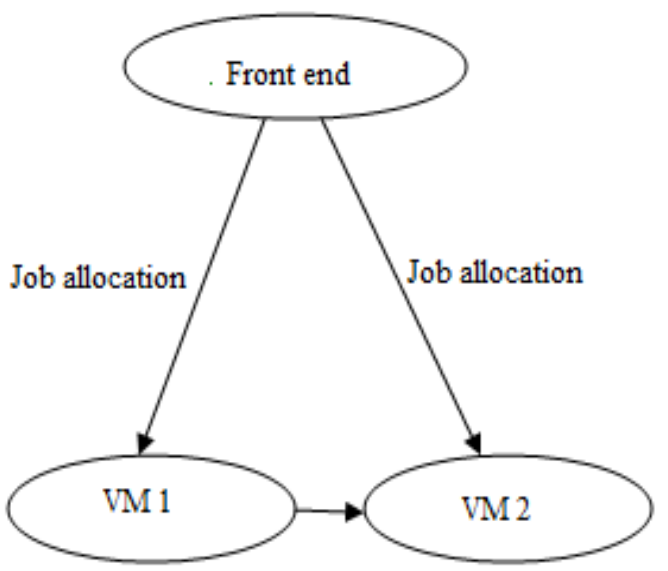

Fig. 1. Architecture of the proposed system.

Fig. 1 shows the architecture of the proposed system. Jobs will be allocated to the two virtual machines from the front end. And the working rate of the two virtual machines will be calculated to allocate the respective jobs.

Cloud services are entirely based on distributed computing, in order to achieve high performance computing [6].

Many Task computing will be implemented in the Multi-cloud Environment. Performance analysis will be carried over in the multi cloud services in order to complete multiple tasks in the MTC Applications. Performance analysis consists of CPU usage, disk utilization, I/O tasks, failure rate of the server and Cost Benefit analysis [6]. The list of CPU jobs will be considered as jobs.

\section{A. Cloud Computing Services for Scientific Computing}

We provide a background to analyze the performance of cloud computing services for scientific computing. We introduce two virtual servers and then describe the working rate of the two virtual servers. Working rate will be calculated on the basis of CPU usage, disk utilization, I/O task, failure rate of the server. Then, we introduce the cloud computing services that can be used for scientific computing.

\section{B. Modules}

The modules are

1. IP address representation module

2. Job description

3. Cluster formation

4. Job allocation and performance analysis

5. Job scheduling

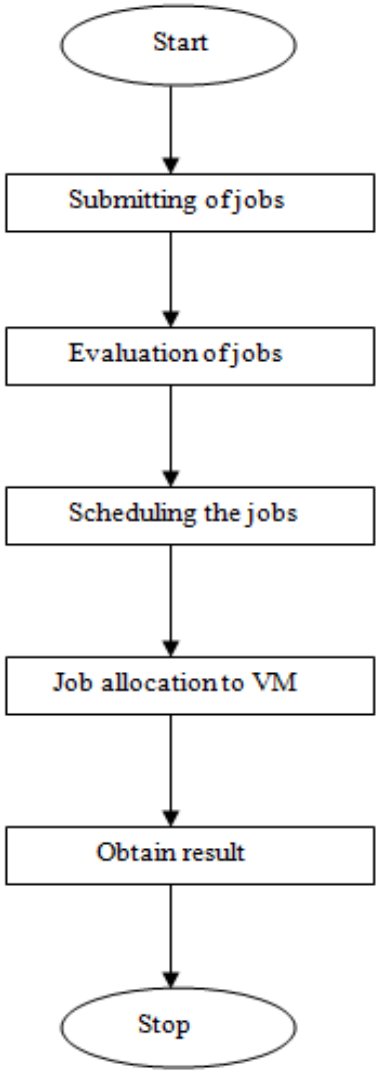

unit 


\section{IP Address representation module:}

The IP Address Representation module is to give the IP addresses which we are going to assign those as servers. We can enter and view IP addresses from this module. The connection has to be represented by the IP Address representation only.

\section{Job description:}

In the Job description, various services will be introduced which could be done by the virtual servers. All Jobs will be executed on the remote server and all jobs are selected by the clients dynamically.

\section{Cluster formation :}

Virtual machines are created on the remote machine. These virtual machines act as the virtual cloud server. The jobs allocated by the clients will be executed in the cloud servers. The comparison among the two servers will be done in a cluster. The working rate of one will be less than the other.

\section{Job allocation and performance analysis:}

The performance of the two virtual servers will be recorded and depending on the load on the two servers jobs will be allocated to them. The allocation of jobs will be done on the basis of CPU usage, disk utilization, I/O task, response time. These virtual machines execute the jobs on the basis of which server has the better working rate. The server will then send the execution time of the jobs executed on the server to the client.

\section{Job Scheduling:}

Jobs are allocated from the client machine. If multiple jobs are to be allocated, then the jobs have to be scheduled before allocation of the virtual machines. So, scheduling algorithm will be implemented in the client machines. The execution time of every job will be obtained based on their execution time scheduling process will be done.

\section{CONCLUSION}

In this paper, we presented job scheduling procedure that try to avoid overloading on a node of the cluster. We have discussed the importance of having the information regarding every virtual server of the cluster. We have successfully achieved our aim to avoid overloading any machine on the cluster, utilize maximum resources on a particular virtual machine thereby decrease race condition for resources and overall runtime of the jobs. The CPU jobs will be performed by the virtual server with the better working rate. Since our algorithm mainly focuses on Task Assignment, it can also be plugged-in in conjunction to a Fair or Capacity scheduler. This would add Fair and Capacity schedulers' job selection features into our algorithm.

\section{FUTURE WORK}

Future work of our research includes finding best runtime of the jobs through machine learning technique and tuning of MapReduce framework with different configuration parameters. We plan to propose efficient usage of resource of the cluster to save energy by using scale up/down algorithms with our resource-aware scheduling to switch on/off the virtual machines/nodes.

\section{REFERENCES}

[1] Hung-Chang Hsiao, Hsueh-Yi Chung, Haiying Shen, and Yu-Chang Chao, "Load Rebalancing for Distributed File Systems in Clouds", IEEE transaction on parallel and distributed system, Vol. 24, No. 5, May 2013.

[2] Alexandru Iosup, Simon Ostermann,Nezih Yigitbasi, Radu Prodan, , Thomas Fahringer, and Dick Epema, "Performance Analysis of Cloud Computing Services for Many-Tasks Scientific Computing”, IEEE TPDS, MANY-TASK COMPUTING, NOVEMBER 2010

[3] K.Senthil, "Performance Analysis of multi-cloud Deployment in Many task Applications", International Journal of Engineering Research \& Technology (IJERT), Vol. 1 Issue 5, July - 2012.

[4] Jeffrey Dean and Sanjay Ghemawat, "MapReduce: Simplified Data Processing on Large Clusters", OSDI 2004.

[5] Daniel Warneke and Odej Kao, "Exploiting Dynamic Resource Allocation for Efficient Parallel Data Processing in the Cloud", IEEE transaction on parallel and distributed system, 2011.

[6] Rafael Moreno-Vozmediano, Ruben S. Montero, Ignacio M. Llorente; "Multi-Cloud Deployment of Computing Clusters for Loosely-Coupled MTC Applications”, IEEE TPDS (SPECIAL ISSUE ON MANY-TASK COMPUTING), JULY 2010. 